\title{
Risk-based optimization of emergency rescue facilities locations for large-scale environmental accidents to improve urban public safety
}

\author{
Ming Zhao • Qiuwen Chen
}

Received: 15 January 2014/ Accepted: 25 June 2014/Published online: 8 July 2014

(C) Springer Science+Business Media Dordrecht 2014

\begin{abstract}
Emergency rescue facility is an indispensable component of urban system and important for public safety. Due to the special characteristics and complexity of urban environmental accidents, locations of emergency rescue facilities should be optimized, and the decision criteria should be comprehensive, taking into account not only the spatial relations of these facilities but also the potential hazard of environmental accidents. This study proposes an innovative methodological framework to optimize the locations of emergency rescue facilities in the contexts of large-scale urban environmental accidents. Risk-based approach is involved to delineate the potential hazard of environmental accidents to the adjacent urban areas, and a three-objective decision optimization model is constructed. Moreover, an appropriate spatial representation and encoding strategy is designed and coupled with the non-dominated sorting genetic algorithm-II for model solving. A case study is presented to demonstrate the methodology, and it provides evidence that the involvement of risk mapping into the model construction has significant effect on the optimization result. The findings show that the strategy proposed in this study has the potential to be a useful decision support tool for urban planning with respect to public safety.
\end{abstract}

Keywords Environmental accidents · Facility location · Risk assessment · Spatial optimization - Urban public safety

\section{Introduction}

Following the rapid industrialization, lots of industrial plants have been set up in the vicinity of urban areas in China due to the convenient transport logistics system and

M. Zhao $(\bowtie) \cdot$ Q. Chen

Research Center for Eco-Environmental Sciences, Chinese Academy of Sciences,

Shuangqing Road 18, Beijing 100085, China

e-mail: nkwindancer@gmail.com 
abundant human resources. In these plants, certain quantities of hazardous materials (toxic, flammable or explosive substances) are unavoidably involved into manufacturing process, which can potentially lead to major environmental accidents such as chemical leakage, fire, explosion or toxic proliferation (Zhou and Liu 2012). When an accident occurs, the impact often spreads to the adjacent urban areas with high-density population, causing catastrophic effects and leading to heavy casualties and property losses (Georgiadou et al. 2010; Li et al. 2010; Huang et al. 2011; Fan 2014). Public safety and health in the highly populated urban areas has thus caught national attentions in China (Xiang et al. 2011).

In order to mitigate the disaster and minimize the damage, some kinds of protective actions usually should be carried out in a short time after the accident occurrence, including the management, allocation and distribution of emergency rescue materials (e.g., shelters, food, water, tablets, protective equipment, etc.) (FEMA 2008). These actions are often implemented by setting up a number of emergency rescue facilities (Jia et al. 2007a, b; Georgiadou et al. 2010; Horner and Downs 2010). Locations of these emergency rescue facilities are crucial for ensuring the efficiency of emergency relief distribution and casualty transportation (FEMA 2008; Lee et al. 2009; Horner and Downs 2010; Maliszewski and Horner 2010; Caunhye et al. 2012; Maliszewski et al. 2012). Although these emergency rescue facilities play a role in the emergency response phase after disasters occurrence, the planning of their locations should be carried out in the emergency preparedness phase before disasters occur. In this context, developing optimal strategies for effectively locating these emergency rescue facilities is of utmost importance for disaster mitigation and public safety protection.

The essential issue of emergency rescue facilities locations (ERFLs) problem is actually a facility location optimization problem (Church and ReVelle 1974; Revelle and Hogan 1989; Jia et al. 2007a; Church and Murray 2009). Research on facility location problem is abundant, and many decision models have been developed to solve various facility location problems, including the ones in the context of regular emergency services (e.g., fire stations, medical centers, etc.) (Chrissis 1980; Revelle and Snyder 1995; Liu et al. 2006; Alçada-Almeida et al. 2009; Indriasari et al. 2010; Maliszewski and Horner 2010), as well as the ones for large-scale disasters emergency services (e.g., earthquakes, floods, hurricanes, etc.) (Jia et al. 2007a, 2007b; Horner and Downs 2010; Bell et al. 2011; Caunhye et al. 2012; Maliszewski et al. 2012). However, models proposed for environmental accidents emergency services are sparse, which are actually much more frequent hazardous events than nature disasters in urban system. Therefore, improving existing ERFLs optimization model to accommodate the special characteristic of environmental accidents emergency has more practical significance in urban areas.

In urban areas, the hazards of environmental accidents to different adjacent areas are distinctly distinguishing due to the spatial pattern of urban system and its natural geographical condition ( $\mathrm{Li}$ et al. 2010). This implies that the criteria for optimizing ERFLs should be comprehensive, taking into account not only the spatial characteristics of these facilities but the potential hazard of environmental accidents. In recent years, different methods have been developed and applied to evaluate the potential hazard of environmental accidents. In general, there exist two kinds: the consequence-based approach and the risk-based approach (Salvi and Debray 2006). The consequence-based approach is based on the assessment of consequences of potential accidents, without explicitly quantifying the likelihood of them (Sebos et al. 2010). A simplified form of the consequencebased approach is the use of "generic" separation distance in which the physical (e.g., toxic concentration) or human health impacts reach with a threshold value in a given exposure period (Kontić and Kontić 2009). In contrast, risk-based approach focuses on the 
assessment of both consequences of potential accidents and expected occurrence probability of these accidents (CPR18E 1999). The risk represents the likelihood that an element located at a fixed site with no protection suffers any hazard (e.g., thermal radiation, overpressure, acute toxicity, etc), and it does not consider whether or not the individual element is actually present (Jonkman et al. 2003; Zhou and Liu 2012). Compared to the consequence-based approach, risk-based approach is more comprehensive and efficient in quantifying the hazard of environmental accidents to adjacent areas, and it has been successfully demonstrated in guiding some kinds of urban planning problems, such as land-use planning (Bottelberghs 2000; Ale 2002; Cozzani et al. 2006; Basta et al. 2007; Sebos et al. 2010; Zhou and Liu 2012). These applications also suggest a promising future for applying the risk-based approaches in solving the ERFLs problem in large-scale urban environmental accidents emergency situations.

Another challenge is that large-scale ERFLs problem unavoidably involves huge sets of geographical data, implying that model's efficient solving is difficult. At present, the approaches proposed for solving large-scale ERFLs problem are generally exact approaches, such as linear, nonlinear or other mathematical programming methods (Jia et al. 2007a; Maliszewski and Horner 2010; Horner and Downs 2010; Maliszewski et al. 2012; Bell et al. 2011). Unfortunately, there are practical limitations associated with the use of exact methods in the large-scale ERFLs problem, as it is actually a geographical optimization problem. Geographical optimization problem is recognized as a typical NP-hard problem, and it is often computationally intensive to solve (Xiao et al. 2002). The computational effort often increases dramatically with extremely numerous sets of spatial data, and the number of feasible solutions may increase exponentially with the problem size (Xiao 2008). This means that medium- to large-sized solution space problems often exceed the capabilities of these exact approaches (Tong et al. 2009; Murray 2010). In order to overcome these limitations of exact methods, some scholars have resorted to a number of heuristic approaches to solve geographical optimization problems effectively and efficiently (Xiao 2008; Tong and Murray 2012). Among various modern heuristic approaches, evolutionary algorithms (e.g., GA) have shown great promise for generating solutions to large-scale ERFLs problem (Jia et al. 2007b; Indriasari et al. 2010; Mohammadi et al. 2010). When GA is applied to solve multi-objective optimization problems, however, the scalar fitness information should be provided to combine multiple objectives into a single objective by using aggregating approaches (e.g., weighted sum approach, goal programming, etc.) (Coello 1999). This strategy is simple in use, but its limitation is obvious especially in solving optimization problems that involve more than two decision objectives. The first is that some accurate scalar information on the range of objectives should be provided, which is normally a very expensive process in most real-world application cases (Coello 1999). Moreover, there is a serious drawback of the weighted sum approach as it may miss concave portions of the trade-off curve, in other words, it may miss important solutions, especially when the Pareto front contains concave and/or discontinuous sections (Coello 1999; Xiao et al. 2002). Furthermore, it is usually difficult for decision makers to determine the appropriate weights when some features of the problem are not fully understood during the early stages of decision making (Miettinen 1999; Xiao et al. 2007). In this context, appropriate approach is needed to solve the multi-objective ERFLs problem in a large-scale emergency situation.

In the last decades, several novel algorithms have emerged in the evolutionary computation community proposed for solving multi-objective optimization problems, called multi-objective evolutionary algorithms (MOEAs) (Zitzler and Thiele 1999; Deb et al. 2002; Knowles et al. 2008). MOEAs are essentially heuristic methods that are often more 
efficient than exact approaches, they are less susceptible to the shape or continuity of the Pareto-optimal frontier (Coello 1999). They are derived from evolutionary algorithms (EAs) and have been successfully demonstrated as efficient posterior approaches in solving various multi-objective spatial optimization problems (Xiao et al. 2002, 2007; Farhana and Murray 2008; Lee and Xiao 2009; Huang et al. 2008; Cao et al. 2011; Wu et al. 2011; Tong and Murray 2012; Bhattacharya and Bandyopadhyay 2010; Lančinskas and Zilinskas 2013). The main advantage of MOEA is their ability to deal simultaneously with a set of possible solutions and allow one to find multiple Pareto-optimal solutions in a single simulation run, instead of having to perform a series of separate runs as in the case of traditional mathematical programming methods (Deb et al. 2002; Georgiadou et al. 2010; Srinivas and Deb 1994). The strategy in MOEA is called posterior articulation of preferences, which does not require the intensive participation of decision makers before or during the process of generating alternatives. A diverse set of nondominated solutions can be generated and distributed on the Pareto-optimal frontier. These solutions are subsequently presented to the decision makers, who make a final decision about the problem by examining and negotiating about the merits of alternatives. These advantages of MOEA suggest a promising future for solving the multi-objective ERFLs problem in large-scale emergency situations, as well as the model's incorporation into a decision support system.

In this study, an innovative methodology framework is proposed for decision makers to cope with the complexity of the ERFLs problem in urban environmental accidents emergency management. The procedure of decision optimization model construction and the strategy for model solution are presented. There are several key features that distinguish our research from previous works. First, risk-based approach is involved to delineate the potential hazard of environmental accidents to the adjacent urban areas. Second, a three-objective decision optimization model is constructed based on the result of risk mapping. And then, an appropriate spatial presentation and encoding strategy is designed and coupled with the NSGA-II algorithm for model solution. A case study is presented to demonstrate the capabilities and potentials of the methodology.

\section{Multi-objective optimization model for ERFLs problem}

The methodological framework developed in this study is shown in Fig. 1. According to the special characteristic of ERFLs problem, a three-objective decision optimization model is first constructed, representing service capacity, global efficiency and equity, respectively. Based on the spatial representation method, risk assessment of the hazard sources are carried out, and the results of risk mapping are imported to construct a risk-based spatial decision optimization model. Then, the model is encoded and MOEA is used for model solution. Finally, a pool of candidate solutions are provided to the decision makers, and they can select suitable solutions in accordance with their own subjective preferences or determinant criteria One key point of the proposed method is that the hazard of environmental accidents is identified, quantified and integrated into a risk-based optimization model.

In this section, the multi-objective decision optimization model for ERFLs problem is constructed based on the special characteristic of environmental accidents emergency. 


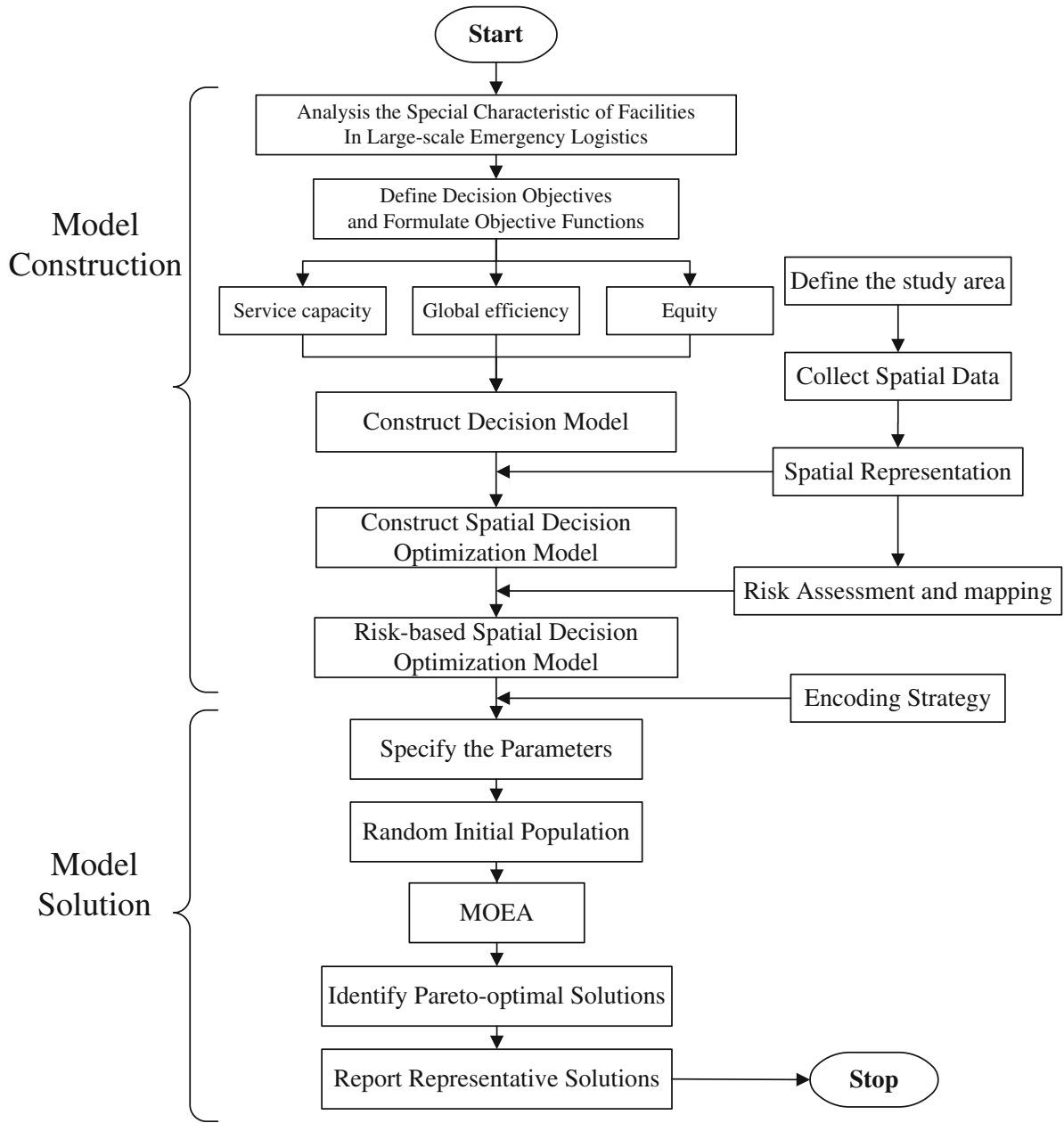

Fig. 1 Flowchart of the methodology for solving ERFLs problem

\subsection{Assumptions}

In order to formulate the ERFLs problem, the following assumptions are made:

1. The entire study area is deemed as the demand region, i.e., the region of interest is represented by a two-dimensional continuous space;

2. The service capacity limitation of each emergency rescue facility is not taken into consideration, i.e., the resources and service abilities of each facility (e.g., the amount of supplies, medical staff, vehicles etc.) are sufficient to support the demand units it services;

3. The service distance of each facility is not constrained, as individual facility may be destroyed by the disaster and resource allocation among different facilities is usually carried out in emergency rescue (FEMA 2008). 


\subsection{Model formulations}

The mathematical specification of the multi-objective optimization model relies on the following notations:

Inputs:

$n \quad$ Set of demand units;

$m \quad$ Set of potential facility sites;

$i \quad$ Index of demand units, $i \in n$;

$j \quad$ Index of potential facility sites, $j \in m$;

$R_{i} \quad$ Potential risk value of demand unit $i, i \in n$;

$p \quad$ Number of facilities to be located;

$d_{i j} \quad$ The distance or travel time between any a demand unit $i$ and each potential facility site $j$. In this study, the Euclidian distance is used and calculated based upon their $x$-coordinates and $y$-coordinates;

$$
d_{i j}=\sqrt{\left(x_{i}-x_{j}\right)^{2}+\left(y_{i}-y_{j}\right)^{2}}
$$

Attenuation coefficient and attenuation factor, respectively, indicating that the $k \& e^{-k d_{i j}} \quad$ service capacity will decrease with increasing spatial distance between the facilities and demand units (Li and Yeh 2005):

$$
a_{i j}=\left\{\begin{array}{l}
1, \text { if facility located at site } j \text { covers demand unit } i \\
0, \text { if otherwise }
\end{array} \forall i \in n, j \in m .\right.
$$

Decision variables:

$L_{\max } \quad$ The maximum distance between any demand unit and its closest facility;

$X_{j}=\left\{\begin{array}{l}1, \text { if a facility is located at potential site } j \quad \forall j \in m ; \\ 0, \text { if otherwise }\end{array}\right.$

$Y_{i}=\left\{\begin{array}{l}1, \text { if demand unit } i \text { is covered by at least one facility } \\ 0, \text { if otherwise }\end{array} \forall i \in n ;\right.$

$Z_{i j}=\left\{\begin{array}{l}1, \text { if demands at unit } i \text { are served by a facility located at site } j \\ 0, \text { if otherwise }\end{array} \forall i \in n, j \in m\right.$.

The model formulation is as follows:

$$
\begin{gathered}
\operatorname{maximize} F_{1}=\sum_{i \in n} \sum_{j \in m} R_{i} Y_{i} e^{-k d_{i j}} \\
\operatorname{minimize} F_{2}=\sum_{i \in n} \sum_{j \in m} R_{i} d_{i j} Z_{i j} \\
\operatorname{minimize} F_{3}=L_{\max }
\end{gathered}
$$

Subject to: 


$$
\begin{gathered}
Y_{i} \leq \sum_{j \in m} a_{i j} X_{j}, \quad \forall i \in n \\
\sum_{j \in m} X_{j}=p, \\
Z_{i j} \leq X_{j}, \quad \forall i \in n, \quad j \in m \\
\sum_{j \in m} Z_{i j}=1, \quad \forall i \in n \\
\sum_{j \in m} d_{i j} Z_{i j}-L_{\max } \leq 0, \quad \forall i \in n \\
X_{j}=\{0,1\} \quad \forall j \in m \\
Y_{i}=\{0,1\} \quad \forall i \in n \\
Z_{i j}=\{0,1\} \quad \forall i \in n, j \in m
\end{gathered}
$$

The model has three objectives, where objective (1) maximizes the amount of demand covered by a specified number of facilities, representing the global rescue capacity is maximum (Church and ReVelle 1974). Its primary purpose is to service the highly risk areas with more facilities. Objective (2) minimizes the sum of total weighted distances between any demand unit and its closest facility (i.e., $p$-median model) (Daskin 1995). It represents the global rescue efficiency of these emergency rescue facilities is maximum, as a facility that is close to a demand unit provides a better quality of emergency rescue service than the facilities located far from that demand unit (Jia et al. 2007b). Objective (3) minimizes the maximum distance $L_{\max }$ or response time from any demand point to its closest facility (i.e., $p$-center model), representing the equity of locating these emergency rescue facilities within the region is maximized (Daskin 1995).

Constraints (4) state that demand at unit $i$ cannot be covered unless at least one of the facility sites that cover unit $i$ is selected. Constraints (5) stipulate that $p$ facilities are to be located. Constraints (6) state that demands at unit $i$ can only be assigned to a facility at location $j\left(Z_{i j}=1\right)$ if a facility is located at node $j\left(X_{j}=1\right)$. Constraints (7) state that each demand unit $i$ must be assigned to a facility at some node $j$. Constraints (8) state that $L_{\max }$ must be greater than the distance between any demand unit $i$ and the open facility $j$ to which it is assigned. Constraints (9), (10) and (11) are the integrality constraints on the decision variables.

The combination of these objective functions and constraints constitute a multi-objective decision optimization model for the ERFLs problem, which is:

$$
\text { minimize }\left[\mathrm{obj}_{1}, \mathrm{obj}_{2}, \mathrm{obj}_{3}\right] \quad \text { where } \mathrm{obj}_{1}=\frac{1}{F_{1}}, \mathrm{obj}_{2}=F_{2}, \mathrm{obj}_{3}=F_{3}
$$

\subsection{Model solution}

MOEAs have been proved as efficient approaches to solve various multi-objective optimization problems, and encoding scheme is important as it has much effect on performance (Tong et al. 2009). In a standard EA, solutions are usually encoded as binary strings or real numbers (Xiao et al. 2002). Unfortunately, these encoding schemes cannot guarantee to produce feasible child solutions and may lead to poor performance, even for very small problems (Tong et al. 2009). Therefore, applying these encoding schemes directly in 


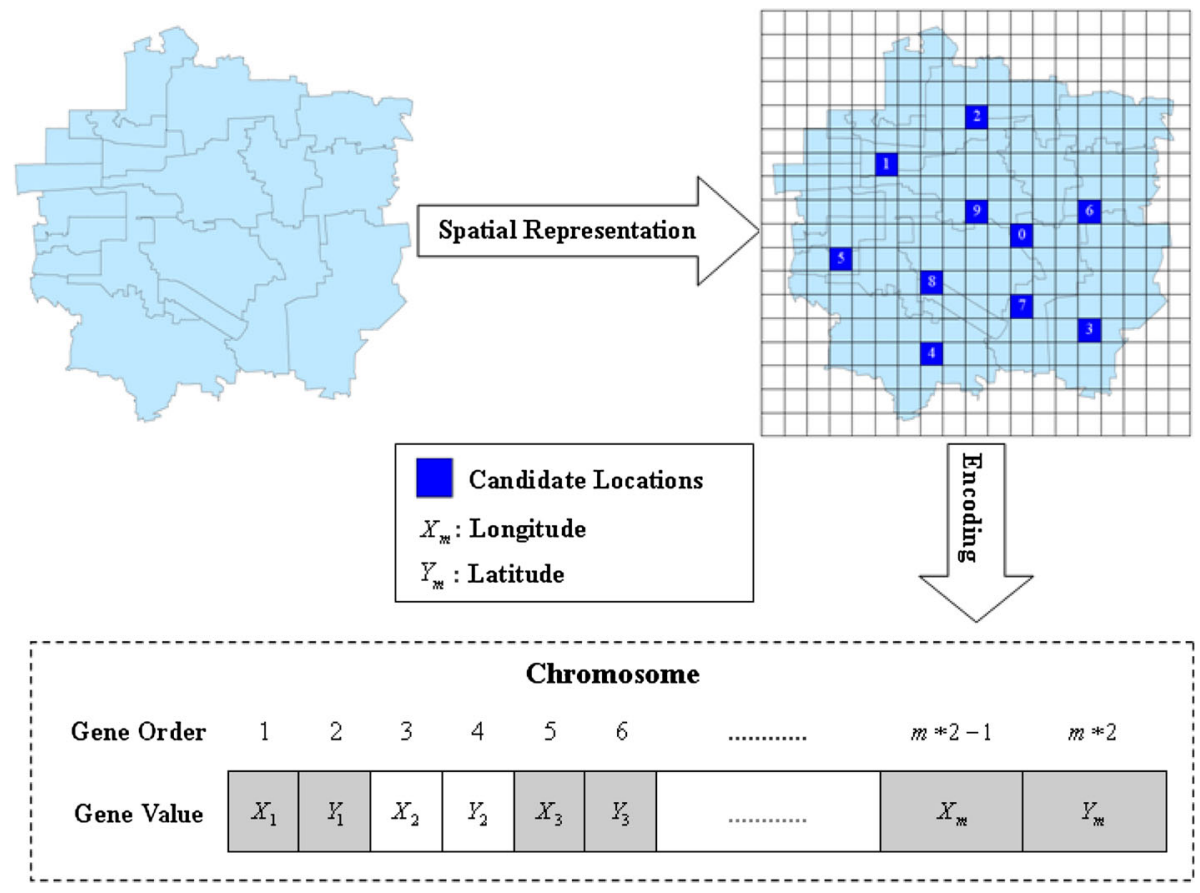

Fig. 2 Spatial representation and encoding strategy designed for model solution

solving large-scale spatial optimization problems (e.g., large-scale ERFLs problem) is intractable due to massive spatial data involved and huge solution space (Tong et al. 2009). In this context, some scholars have tried to design some kinds of "natural" representation and encoding schemes that are suitable for their problems being addressed, such as graph theory-based representation strategy (Xiao et al. 2002, 2007; Xiao 2008) or integer vector encoding scheme (Jia et al. 2007b; Tong et al. 2009). These "natural" schemes conveniently encode solutions of spatial optimization problems in an EA and have been successfully demonstrated as efficient encoding schemes in solving various large-scale geographical optimization problems (Jia et al. 2007b; Xiao et al. 2007; Tong et al. 2009). In this study, a mesh-based spatial representation strategy is adopted for the convenience of genetic coding and the accuracy improvement of search results. Specifically, the entire study region is divided into a number of fine spatial meshes, shown in Fig. 2. Each mesh represents a demand unit, and the total number of meshes in the area is equal to $\mathrm{M} \times \mathrm{N}$, where $\mathrm{M}$ and $\mathrm{N}$ are the total numbers of rows and columns. Subsequently, risk value is assigned to each mesh, corresponding to the weight value $R_{i}$ of objective function (1) and (2). Consequently, the spatial location of each mesh can be represented by its $x$-coordinate and $y$-coordinate.

Moreover, an appropriate genetic encoding scheme, combining $\mathrm{x}$-coordinate and $\mathrm{y}$-coordinate of each potential facility, is designed for model solving based on the basic principles of EA, as shown in Fig. 2. The encoding scheme is similar to the integer vector encoding scheme which has recently been used in solving various spatial optimization problems (Jia et al. 2007b; Tong et al. 2009). Specifically, the decision space is the same as the set of entire meshes, i.e., the locations of candidate facilities are selected from the 
entire meshes in the region. Then the $x$-coordinate and $y$-coordinate of each candidate facility $P_{\mathrm{m}}\left(X_{\mathrm{m}}, Y_{\mathrm{m}}\right)$ are defined as a "gene"; the "chromosome" $G$ is then encoded sequentially by the coordinates of each candidate facility $P_{\mathrm{m}}\left(X_{\mathrm{m}}, Y_{\mathrm{m}}\right)$ in the following form:

$$
G\left(P_{1}, P_{2}, \ldots, P_{m}\right),
$$

where $m$ is the number of facilities that will be set up in the region, which is the same as the decision variables.

By using the spatial representation and encoding strategy, the multi-objective optimization model can be coupled with MOEA for model solution. In this study, a well-known and efficient MOEA, NSGA-II, is used for solving the multi-objective ERFLs problem. NSGA-II involves an elitist-preserving approach to speed up the performance of the algorithm and help prevent the loss of elitist solutions once they are found. Moreover, a fast non-dominated sorting approach is applied to reduce the computational complexity, and a crowding distance evaluation mechanism is used to preserve the diversification of Pareto-optimal solutions. For more details of the algorithm, please refer to the literature (Deb et al. 2002).

\section{Risk assessment and mapping}

The potential hazard of environmental accidents to the adjacent urban areas is assessed with three main component elements described in Fig. 3: hazard source (e.g., a liquid chlorine tank, a hydrocarbon tank, etc.), propagation of the effects in the environment (e.g., toxic cloud dispersion, thermal radiation, overpressure of explosion, etc.) and risk targets (Tena-Chollet et al. 2013). Compared to fire ball and explosion accidents, toxic release accidents usually cause much wider hazard zones, and it thus can be more able to reflect

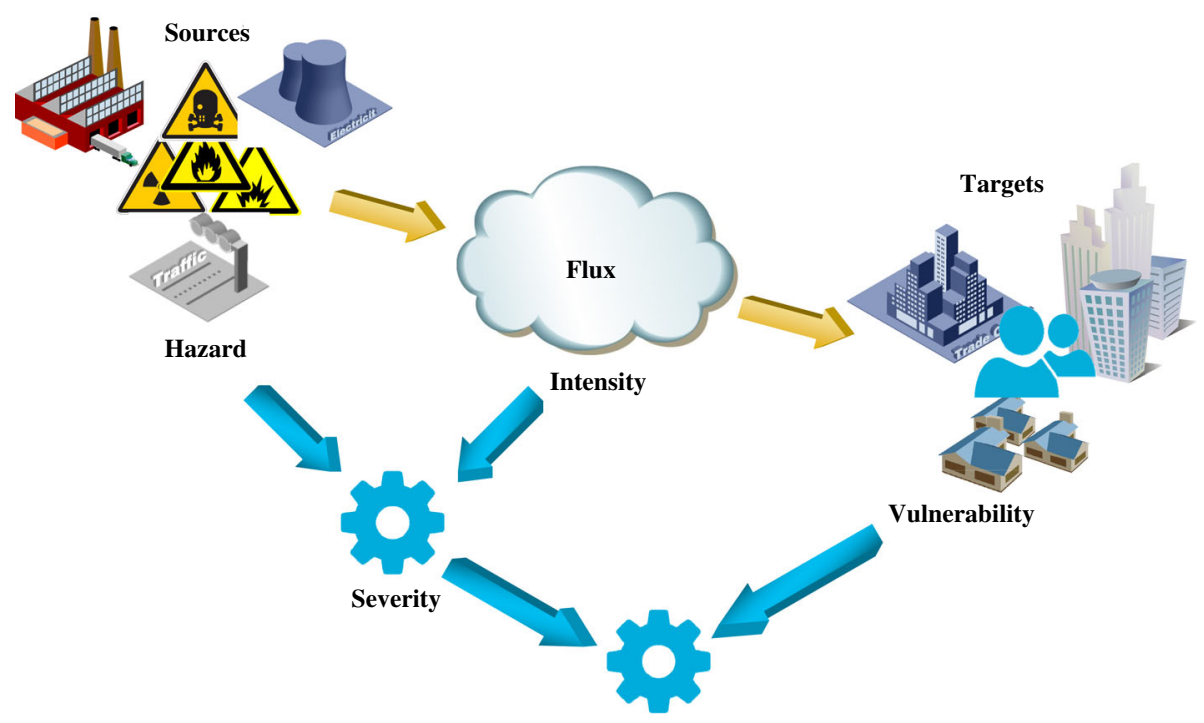

Risk

Fig. 3 Schematization of the main components in risk assessment 


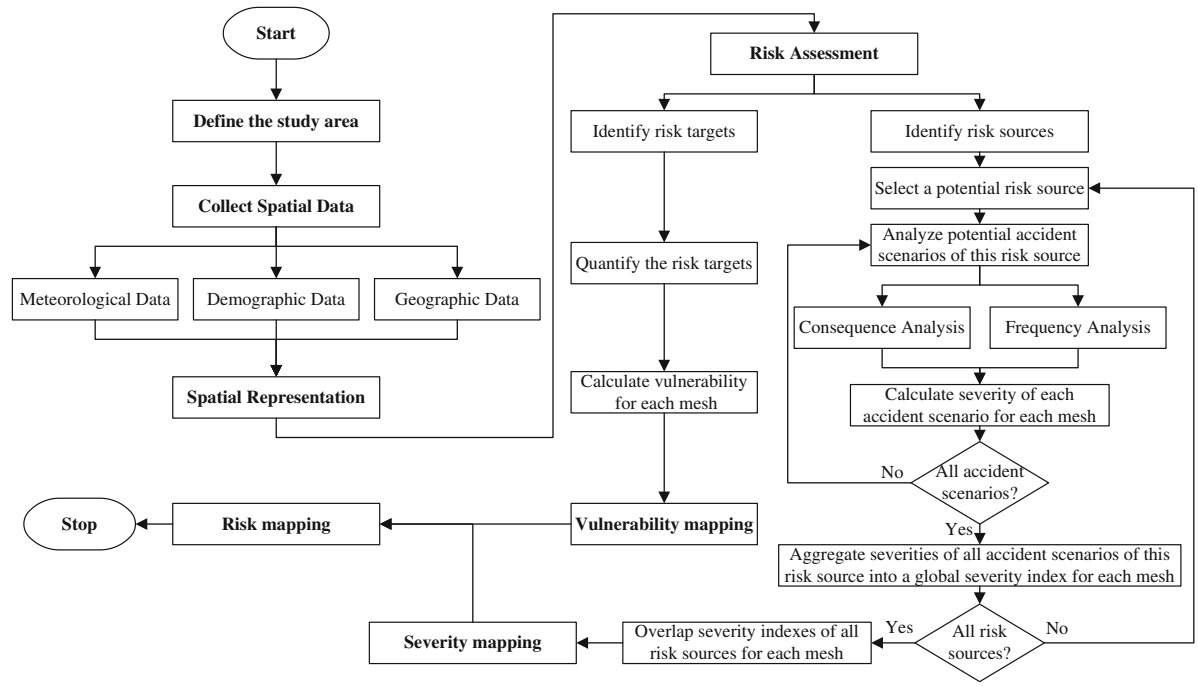

Fig. 4 Flowchart of risk assessment and mapping

the potential hazards to adjacent urban areas. In this study, only toxic gas cloud dangerous phenomenon is considered in the procedure of risk assessment.

The risk is not solely dependent on the proximity and the magnitude of the hazard source, but also on socioeconomic properties of the risk targets (Salvi and Debray 2006; Tixier et al. 2006). Risk assessment requires identifying potential hazard sources that might threaten human targets as well as the capacity of human targets for resisting accidents (Tixier et al. 2006). Therefore, the procedure of risk assessment should be revealed in a synthetic manner, considering both hazard sources and risk targets ( $\mathrm{Li}$ et al. 2010). The process of risk assessment is illustrated in Fig. 4. In principle, it is comprised of four major steps, explained in more details as follows.

\subsection{Generalities}

The features of the study area, as well as the scope and boundary of risk assessment, should be defined. Based on the spatial representation method presented in Sect. 2.3, the mesh size is determined appropriately to keep homogeneity within a mesh and heterogeneity among different meshes (Tixier et al. 2006). Moreover, the procedure of hazard sources identification is carried out according to the relative national standard (AQSIQ 2009). For each mesh, the indexes are calculated sequentially: the severity index, then the vulnerability index and finally the risk level index. The risk level of each mesh can be mathematically expressed as follows:

$$
\text { Risk }=f(\text { severity, vulnerability })
$$

The results of these indexes are then visually shown on the map for easily understanding their contribution to the final risk level.

\subsection{Severity index mapping}

The estimation of severity measures the probability of exposure of sensitive targets to the chemical agents (Li et al. 2010). In this context, it includes two dimensions: intensity of 
Table 1 Thresholds of diverse levels of consequence intensity

\begin{tabular}{ll}
\hline Level of consequence intensity & $\begin{array}{l}\text { Concentration } \\
(C, 60 \text { min exposure })\end{array}$ \\
\hline 1 & AEGL-1 \\
2 & AEGL-2 \\
3 & AEGL-3 \\
\hline
\end{tabular}

Table 2 Thresholds of diverse levels of accident occurrence rate

\begin{tabular}{ll}
\hline $\begin{array}{l}\text { Level of accident occurrence } \\
\text { rate }\end{array}$ & $\begin{array}{l}\text { Range of accident occurrence } \\
\text { rate }(f)\end{array}$ \\
\hline 1 & $f<10^{-8}$ \\
2 & $10^{-8}<f<10^{-7}$ \\
3 & $10^{-7}<f<10^{-6}$ \\
4 & $10^{-6}<f<10^{-5}$ \\
5 & $f>10^{-5}$ \\
\hline
\end{tabular}

accident consequences and frequency of accidents, the former is deterministic and the latter is probabilistic (Planas et al. 2006; Tena-Chollet et al. 2013).

The sensitivity of the targets to the chemical agents represents a dose-effect relationship between them ( $\mathrm{Li}$ et al. 2010). There are several numerical models that can be used to predict the intensity of accident consequence and demarcate the hazard zones within which the hazard level exceed the proposed toxicity thresholds at some time after a release begins. As commonly recommended in risk assessment studies, the Aerial Location of Hazardous Atmospheres (ALOHA) program is employed to predict the intensity of consequence (EPA and NOAA 2007; Zhou and Liu 2012) and Acute Exposure Guideline Levels (AEGLs) are involved to specify the concentration ranges of each threat zone in this study (AEGL 2009). Three categories of threat zones can be identified by plotting concentration isoclines with the three-tiered AEGLs for toxicity thresholds. In this context, weight values are assigned as the consequence intensity of the three-tiered threat zones according to Table 1, the remaining areas beyond these threat zones are treated as 0 .

In order to finalize the severity index, it is necessary to incorporate the accident frequency dimension. For airborne risks posed by environmental accidents (e.g., toxic gas releases), the accident frequency index $\left(F_{\text {accident }}\right)$ is based on the accident occurrence rate $\left(f_{\text {accident }}\right)$ and the statistical frequency of wind direction $\left(P_{\text {wind }}\right)$ in the study area. The thresholds of accident occurrence rate levels are shown in Table 2, and accident frequency index is calculated as follows (CPR18E 1999):

$$
F_{\text {accident }}=f_{\text {accident }} \times P_{\text {wind }}
$$

The specific risk severity index $S_{i}$ of each mesh is defined as the combination of the accident frequency with the accident consequence intensity of each mesh (CPR18E 1999; Planas et al. 2006; Salvi and Debray 2006; Tena-Chollet et al. 2013), i.e.,

$$
S_{i}=\sum_{j \in m} f_{j} \cdot P_{i j} \cdot C_{i j}, \quad i \in n
$$

where 
Table 3 Levels of effects considered

\begin{tabular}{lll}
\hline Level of $S$ & Range of $S$ & Description \\
\hline Low & $<45$ & Small or non-effects \\
Medium & {$[45,75]$} & Reversible effects \\
High & {$[76,105]$} & Irreversible effects \\
Extremely high & $>105$ & Start of lethality or domino effects \\
\hline
\end{tabular}

$n$ : set of the meshes in the study area, $m$ : set of hazard sources in the study area, $i$ : index of mesh, $i \in n, j$ : index of hazard source, $j \in m, f_{j}$ : the occurrence probability of toxic release accident in hazard source $j, P_{i j}$ : the statistical probability of wind direction occurred in mesh $i$ associated to hazard source $j, C_{i j}$ : the consequence intensity level of toxic release accident in mesh $i$ associated to the hazard source $j, S_{i}$ : the severity index of mesh $i$ associated to all of the hazard sources in the study area.

The severity index is then classified into four levels of effects shown in Table 3, which is a representative decision criterion taken to be considered.

\subsection{Vulnerability index mapping}

Vulnerability is the degree of loss to a given element at risk, resulting from the occurrence of a hazard phenomenon with a given magnitude (Tixier et al. 2006). To achieve this goal, the critical or sensitive risk targets within the study area must be identified, located and weighted to assess their relative importance (Tena-Chollet et al. 2013). Moreover, social indicators play a significant role in determining vulnerability, and it is a key point to integrate them into vulnerability assessment (Cutter et al. 2000; Li et al. 2010). According to recent risk assessment studies, some relevant indicators are identified to be pivotal for vulnerability in industrializing countries to major environmental accidents, such as population and its structure, the accessibility of emergency resources, etc. (Cutter et al. 2000; Li et al. 2010). Generally, an area with higher population density and weak accessibility of emergency resources will pose much more risk than other areas (Lara-Valencia et al. 2009). Moreover, critical risk targets should be identified and weighted, such as hospitals, schools, and commercial centers. Therefore, the factors and variables listed in Table 4 are used to characterize the vulnerability of each mesh in this study. The social vulnerability $(V)$ is then measured as follows,

$$
V=\prod_{m=1}^{3} v_{m},
$$

where $v_{m}$ is the indicator which contribute to the vulnerability.

\subsection{Integrating severity and vulnerability into risk}

In this study, both the severity index and the vulnerability index are treated equally and assumed to have the same relative importance in their contributions to the risk. The risk is then calculated as follows:

$$
R_{i}=S_{i} * V_{i}, \quad i \in n
$$

If multiple hazard sources exist, the risk values of each mesh associated to each hazard source are accumulated as the total risk value of that mesh. The risk mapping is 


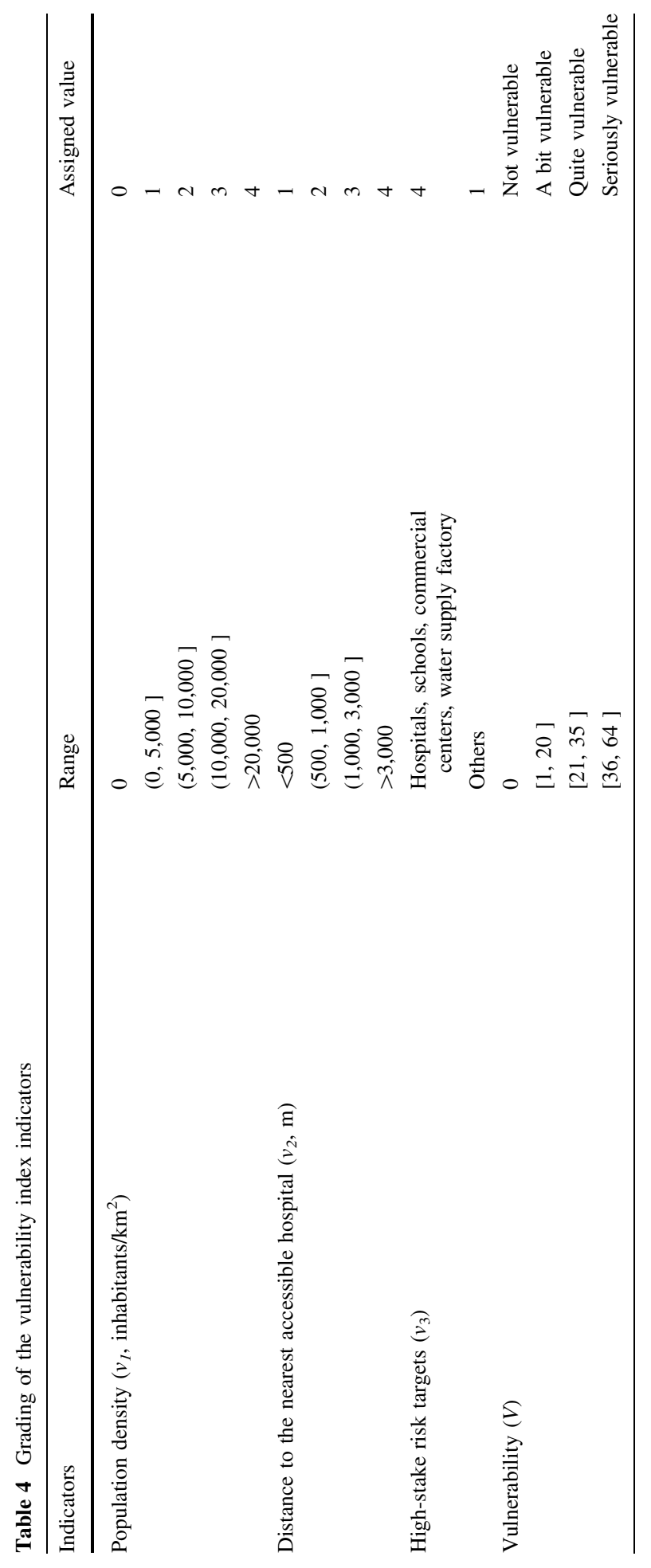


subsequently carried out by overlapping severity and vulnerability in GIS, and the result is integrated into the multi-objective optimization model proposed above.

\section{Case study}

\subsection{Study area}

A typical urban district of northern China, with dense population and many public infrastructures, is selected as the study area to demonstrate the methodology proposed in this study. Spatial geographic data and census data are collected from the local authorities and verified through field survey. The mesh-based population density map is exported and shown in Fig. 6 (a). Moreover, wind rose is derived from historical meteorological data and used to represent meteorological condition of the study area, shown in Fig. 5. Terrain of the study area is plain, thus the effect of terrain on the model's performance could be eliminated.

A thermal power plant, located in the center of the study area, is identified as the primary hazard source shown in Fig. 6a. As it is sensitive and confidential, the detailed information about the major hazard source is fictive in this case study, and any similarity to actual situation is purely coincidental. Specifically, a $90 \mathrm{~m}^{3}$ cylindrical tank filled in $70 \%$ with liquefied ammonia at $2.2 \mathrm{MPa}$ and $298 \mathrm{~K}$ is hypothetically installed in the thermal power plant, and the total amount of mass in the tank is about 42.5 tons. Liquefied ammonia is colorless liquid with characteristic pungent smell, and is both caustic and hazardous; it is widely used in the selective catalytic reduction denitrification process of flue gas in thermal power plants. Liquefied ammonia leakages are common industrial accidents in urban regions of industrializing countries, posing acute toxicity to the local populations. In recent years, many liquefied ammonia industrial accidents have occurred in China, such as the leakage accident occurred in shanghai August 31, 2013, which has caused 15 deaths and many seriously injured.

For the worst damage of the tank, the maximum upper boundary of leakage breach $(0.05 \mathrm{~m})$ is used in the risk calculation (Jian-wei et al. 2012). Due to few statistics of industrial accident probabilities available by far in China, the toxic gas leakage occurrence probabilities of liquid ammonia tank is obtained from the Failure Rate and Event Data (FRED) database in this study (Ale 2002).

\subsection{Model configuration}

Generally, the number of emergency rescue facilities that would be set up is usually limited due to the resource or financial limitations. Therefore, in this case study, the number of candidate emergency rescue facilities is set to 10 , i.e., $P=10$. Based on the strategy presented in Fig. 2, chromosome is encoded to represent the optimal locations of the 10 candidate facilities. Moreover, in Fig. 6a, the number of rows is 101 (i.e., $M=101$ ) and the number of columns is 124 (i.e., $N=124$ ), so the total number of meshes in the area is $M \times N=12,524$. Therefore, the number of candidate solutions in this case study is $C_{12524}^{10}$.

As the parameters setting is very important for algorithm to converge to the optimal, we specify the parameters for the NSGA-II algorithm to be as much similar as the original work (Deb et al. 2002). Specifically, the maximum number of evolutionary generations is 100 , the size of initial population is limited to 100 , the crossover probability is 0.8 and mutation probability is 0.05 . 

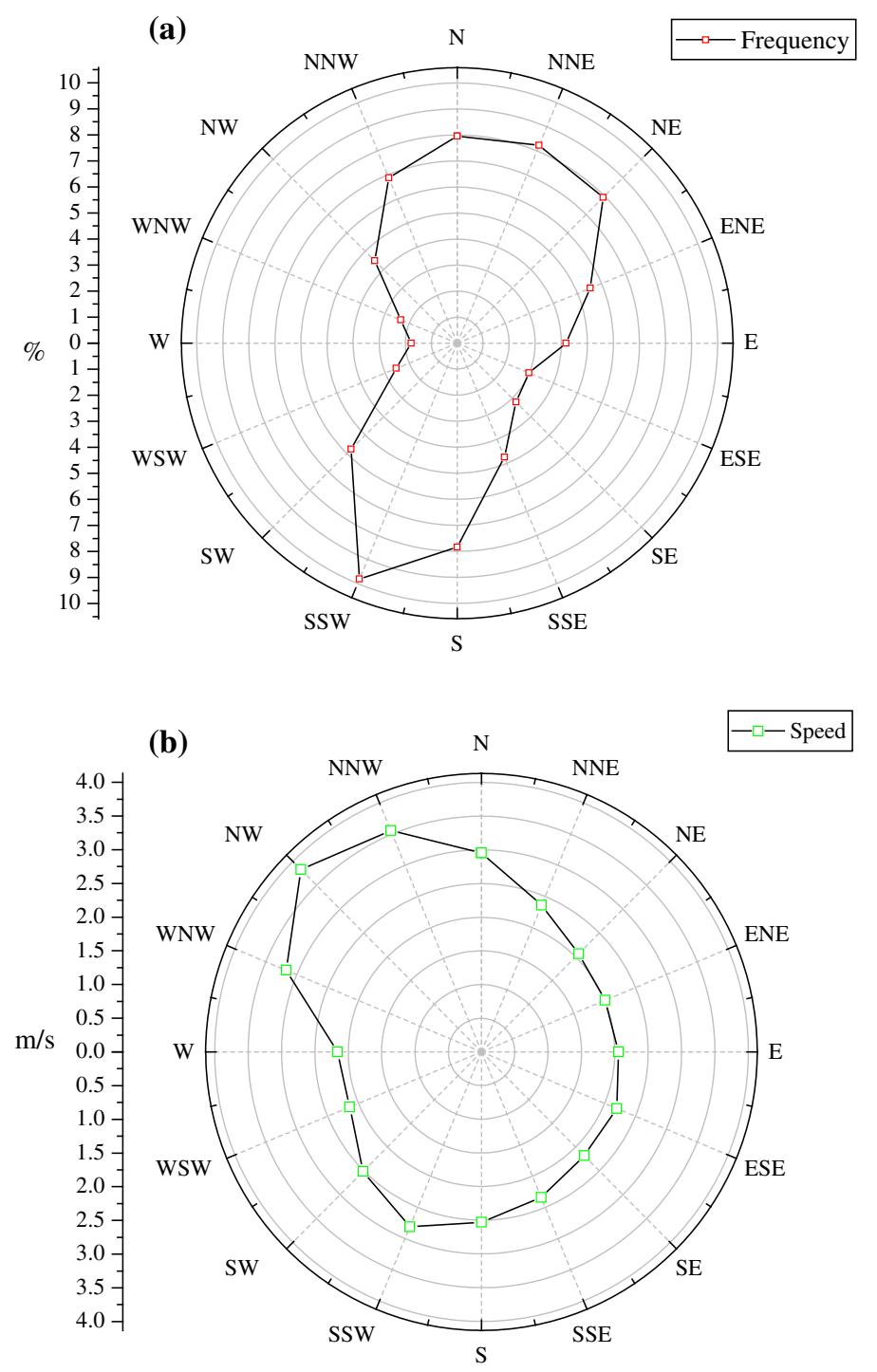

Fig. 5 Wind rose map of the study area: a the frequency of each wind direction; $\mathbf{b}$ the average speed of each wind direction

\section{Results}

\subsection{Risk mapping}

Base on the procedure presented in Sect. 3, the risk severity index of the toxic gas cloud hazard has been calculated and shown in Fig. 6b. According to Table 3, we can find that the severity index values of the study area are distinct: first, in the 1-km areas around the tank, the $S$ values are "Extremely high" in the south and north, "High" in the west, but "Low" in the east; second, in the 1- to 5-km areas around the tank, the $S$ values are "High" 


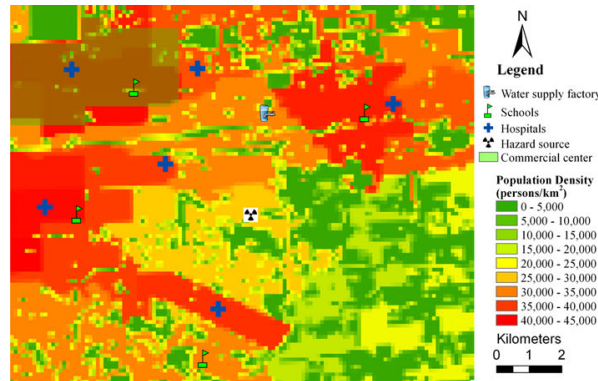

(a)

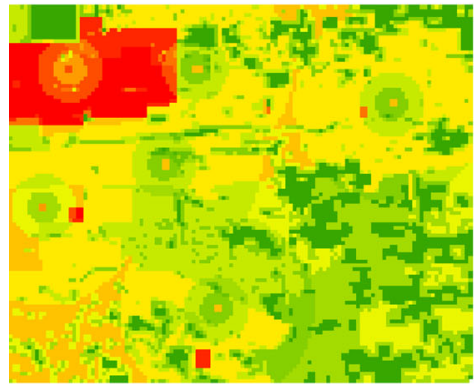

(c)

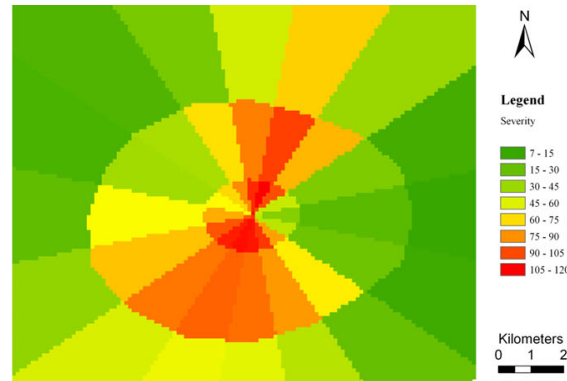

(b)

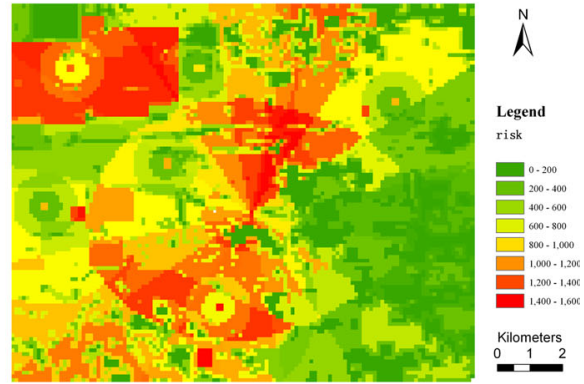

(d)

Fig. 6 Results of mapping: a population density mapping; b severity mapping; c vulnerability mapping; d risk mapping

in the south and north, "Medium" in the west, but still "Low" in the east; finally, outside the 5-km areas around the tank, the $S$ values are relatively "Low" in the east and west, but "Medium" in the north and south.

The vulnerability index has also been calculated and shown in Fig. 6c. On the whole, the lower right part presents "not vulnerable" or "a bit vulnerable," the upper right and the left half parts present "quite vulnerable." Due to the high weight value assigned, the critical risk targets present are "seriously vulnerable." Moreover, there is a confusing phenomenon arising about the meshes in the surrounding of the hospitals. Generally speaking, the vulnerability value of these meshes should be relatively high due to the relatively high population density, but in Fig. 6c, the vulnerability values of these meshes significantly reduce from outside to inside in the form of concentric circles.

Then, the severity and vulnerability indices have been overlapped to produce the actual risk map, shown in Fig. 6d. We can find that the northern and southern areas around the hazard source are with high risk level, because of the relatively higher severity of these areas; the northwest part of the study area is also with high risk level, as the commercial center is located there, which is seriously vulnerable. Moreover, the southwest part of the study area is with medium risk level due to medium severity and vulnerability. The southeast part of the study area is with low risk level as both severity and vulnerability of these meshes are low. 


\subsection{Risk-based ERFLs optimization}

Based on the results above, risk-based multi-objective ERFLs optimization model is constructed and run on a laptop with Intel Core Duo i5 2.60 GHz CPU and 2.0 GB RAM. Due to the stochastic nature of evolutionary algorithm, it is necessary to perform several parallel runs to evaluate its performance (Coello 2005). Without loss of generality, 10 independent runs are implemented by using fixed parameter values but with different random seed generators. For each run, statistical analyses on the final Pareto-optimal solutions are presented in Table 6 of Appendix. The result of experimental run 7 (italic font in Table 6) is deemed as the best, with the fact that a larger number of non-dominated solutions (769) are obtained and the "Sum" is less than that in the other experimental runs. Therefore, the non-dominated solutions obtained from experimental run 7 are taken to draw the final Pareto-optimal frontier, as shown in Fig. 7.

In Fig. 7, the progress of iterations is also shown: the green-cube points represent the initialized population by the random seed operator; the red-ball and blue-pyramid points represent the local Pareto-optimal frontier after the 10th and 50th generation, respectively; the yellow-ball points are the final Pareto-optimal frontier after the 100th generation.

Furthermore, in order to evaluate the capabilities for maintaining diversity and converging progressively to the final Pareto-optimal frontier, we analyzed the behavior of the algorithm during the evolutionary process. From generation to generation, a number of non-dominated solutions emerged, some of which finally form the final Pareto-optimal frontier at the end of 10,000 iterations. The number of non-dominated solutions emerging during every ten generations increases sharply, as shown in Fig. 8a. This indicated that the diversity of non-dominated solutions increased along with the evolutionary process.

Moreover, we analyzed the convergence property of the algorithm based on the convergence metric. In the study, the final Pareto-optimal frontier of the 100th generation is set as the reference, and then, local Pareto-optimal frontiers of the 5th and every 10th generation are compared to it. The result of convergence comparison is shown in Fig. 8b.

After model verification, the 769 non-dominated solutions on the final Pareto-optimal frontier of run 7 constitute a candidate pool for decision makers, and they can then be used to derive suitable trade-off solutions according to the subjective preference of different decision makers. However, there appears to be no best among these non-dominated solutions, as they cannot dominate each other by definition. In order to make a decision among them, decision makers may have their own subjective preferences or determinant criteria. In this study, an equally weighted solution and three extreme preferred solutions are selected among the 769 non-dominated solutions and compared as a demonstration of the model's effectiveness. The four kinds of solutions are respectively labeled as "A," "B," "C" and "Trade-off," shown in Fig. 9.

The location maps of the four solutions are shown in Fig. 10. It is seen that the locations of 10 candidate facilities of solution "A," which is preferred by Obj1, are obviously concentrated around the centroid of the risk map. The locations of 10 candidate facilities of solution "C," which is preferred by Obj3, tend to be quite dispersed comparing to solution "A." The locations of 10 candidate facilities of solution "B," which is preferred by Obj2, are less dispersed than solution " $\mathrm{C}$," but they tend to cover the relatively high-risk regions in order to maximize the global efficiency. The result of the equally weighted solution, which is labeled "Trade-off," has the most balanced or best compromise of service capacity, total global efficiency and equity among all the 769 non-dominated solutions. 


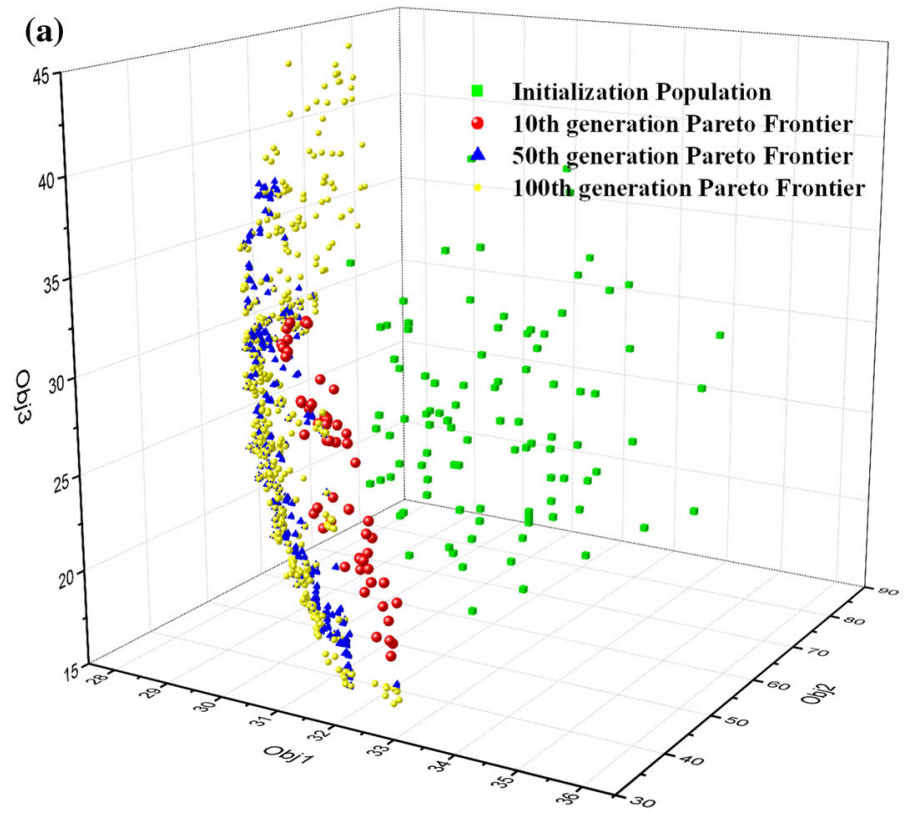

(b)

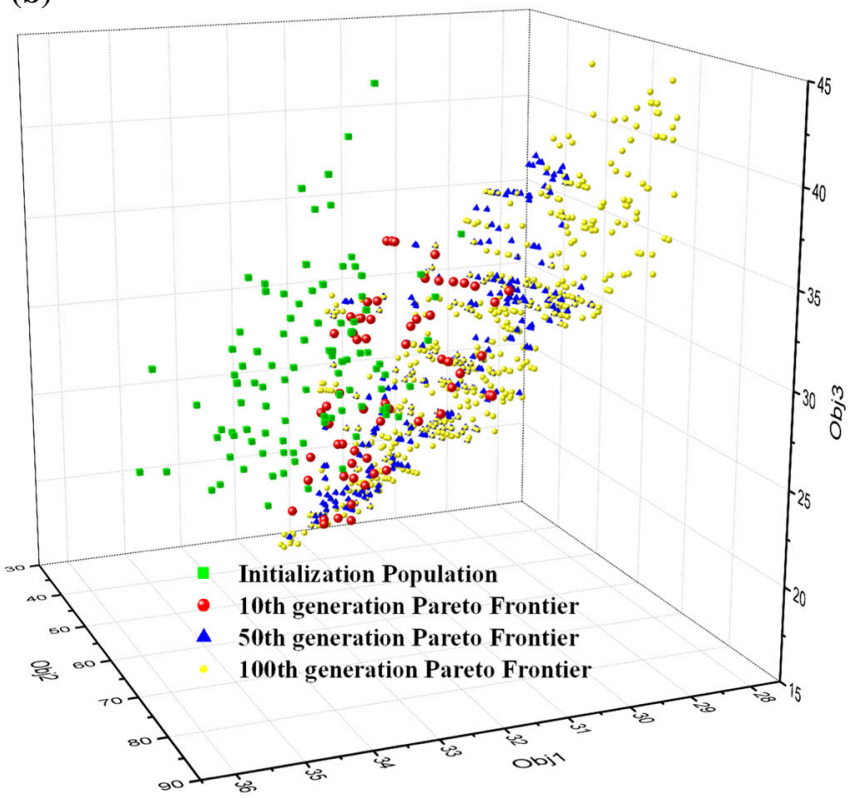

Fig. 7 Pareto-optimal frontiers of different evolutionary generations (Initialization, 10th, 50th, and 100th) representing the performance of a convergence and $\mathbf{b}$ diversity of non-dominated solutions during the process of iterations

Besides the location maps shown in Fig. 10, the attribute information associated with the four kinds of solutions is presented in Table 5. The lowest value (bold font) means the most preferred solution, e.g., Obj1 prefers the solution "A." 
(a)

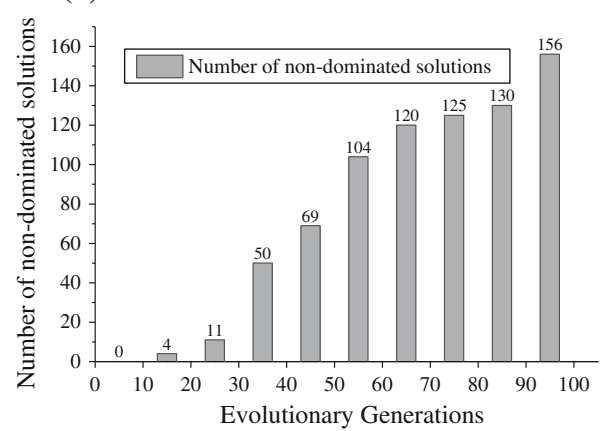

(b)

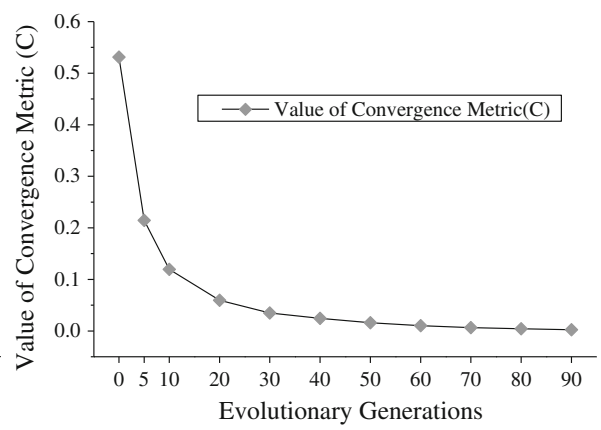

Fig. 8 a The numbers of non-dominated solutions emerging during every ten generations that finally formed the final Pareto-optimal frontier of run 7; $\mathbf{b}$ values of convergence metric of initial population, 5th and every 10 th generation

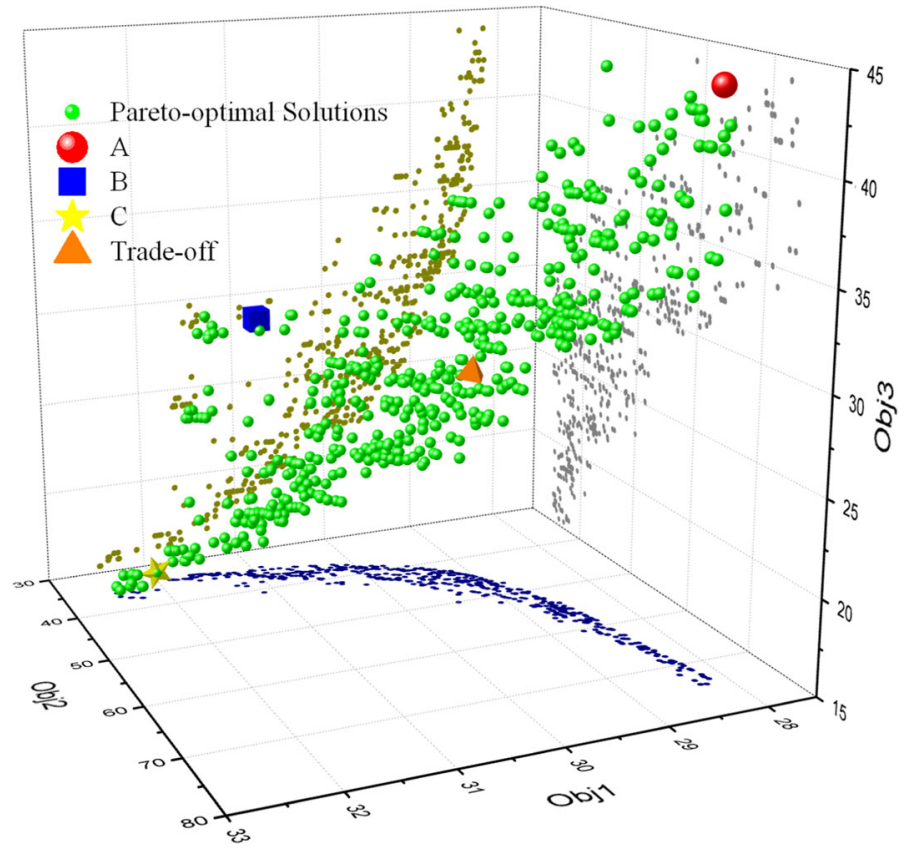

Fig. 9 Three extreme and one trade-off solutions selected among the 769 non-dominated solutions

\subsection{Compare the results of risk-based model with population-based model}

Location map of the "Trade-Off" solution is extracted from Fig. 10 and separately shown in Fig. 11a. We can find that the candidate facilities almost locate at the relatively high-risk areas, no facility locates in the right half part of the area that is with relatively lower risk level. For comparison, a population-based multi-objective optimization model is also constructed by using population density value in place of the variable $R_{i}$ in the decision 

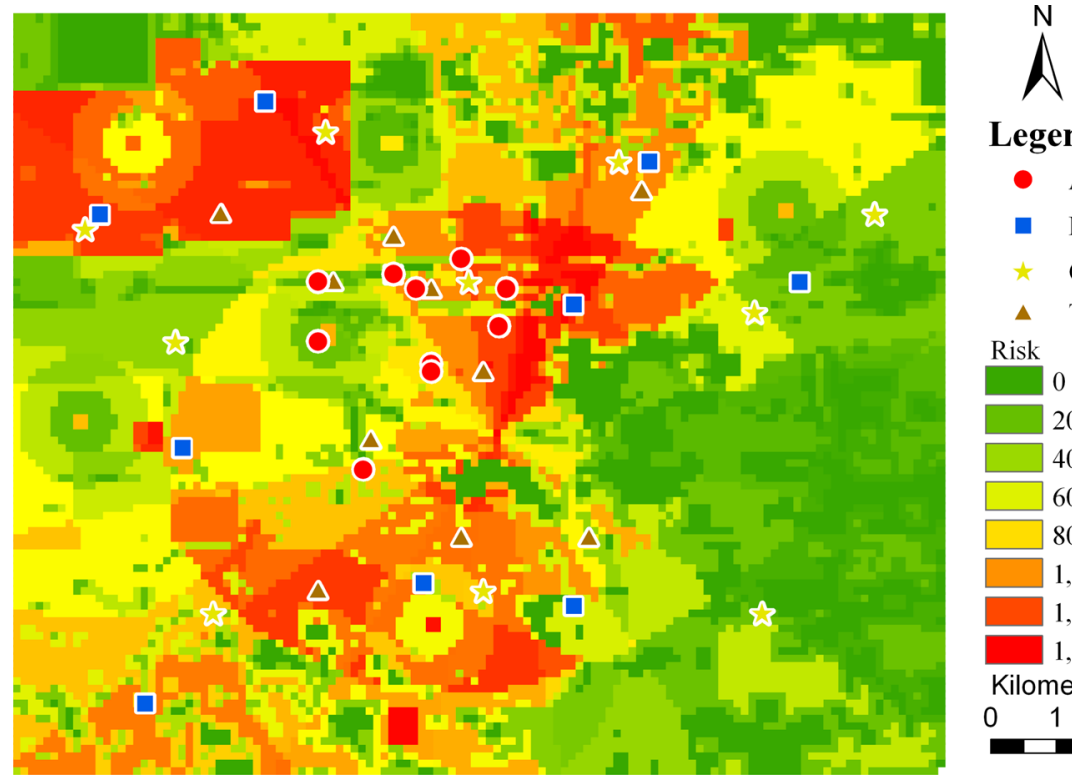

\section{Legend}

- A

- B

* $\mathrm{C}$

$\Delta$ Trade-Off

Risk

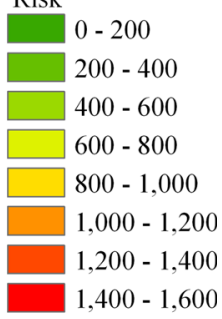

Kilometers

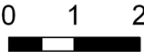

Fig. 10 Location maps of the four representative solutions

model. Then 10 independent experimental runs are also implemented, and statistical analysis of this 10 runs is carried out. A representative trade-off solution named "TradeOff" is selected from the final Pareto-optimal frontier of the "best" run. The location map of non-dominated solution "Trade-Off" is shown in Fig. 11b. We can find that the emergency rescue facilities tend to locate at the populous areas, and only one facility locates at the edge of the lower right part that is with sparse population density.

\section{Discussion}

Figure 5a implies that the predominant wind direction of the study area is north wind (NNE, N, NE and NNW) and south wind (SSW, SW and S). Compare it with Fig. 6b, we can find that the wind rose has significant impact on the atmospheric dispersion as well as the distribution of high-severity areas.

The comparison of Fig. 6a with c clearly depicts that the spatial pattern of vulnerability appears to be similar to the pattern of population density, in other words, meshes with relatively high population density are usually classified as the more serious vulnerable areas. Moreover, the confusing phenomenon arising in the surrounding of the hospitals can be interpreted as the effect involved by indicator $v_{2}$ of Table 4 . Through comparison and analysis, we can find that even these meshes are with relatively high population density; however, the distances from these meshes to the nearest accessible hospital $\left(v_{2}\right)$ are still shorter. Therefore, the rescue services from the nearest hospital to these meshes are relatively much more efficient, and this effect offsets the vulnerability of these meshes to certain extent. Based upon this explanation, another confused question may be emerging, that is why the risk values of the hospitals are still high as the $v_{2}$ of them are zero? This is mainly because of the high-risk values $\left(v_{3}\right)$ assigned to these hospitals. Although the rescue 
Table 5 Attribute information associated with the four kinds of solutions

\begin{tabular}{llll}
\hline Category & Obj1 & Obj2 & Obj3 \\
\hline A & $\mathbf{2 8 . 1 7 3 6 6}$ & 72.48337 & 43.9374 \\
B & 30.90401 & $\mathbf{3 4 . 6 4 1 1 1}$ & 29.24691 \\
C & 32.07267 & 36.31505 & $\mathbf{1 6 . 0 2 0 7 5}$ \\
Trade-Off & 29.14043 & 46.87055 & 26.9931 \\
\hline
\end{tabular}

services to the patients in the hospitals are relatively most efficient, the weak health state of the patients determines they are still high-risk targets as susceptible population.

The comparison of Fig. 6b-d distinctly reflects that the severity and vulnerability index have significant influence on the total risk index. Most of the meshes with serious vulnerability or severity values are finally with high potential risk for the residents lived there. Moreover, through comparing Fig. 6a, d, it is clearly demonstrated that the areas with more residents lived in are not always with the highest risk. In contrast, the areas with lower population density may not be lower risk. It is a critical finding because it reflects the potential "exposure" of residents to the hazard, as well as the likely "rescue costs" for the residents in these areas. On the other hand, it also revealed a fact that the risk mapping has the potential for guiding emergency management. The results produce a broad view of the spatial distribution of total risk within the study area, which provides a new and comprehensive perspective for the decision makers in emergency management community. Thus, some risk avoidance strategies should be taken into account, e.g., allocating more rescue services for the high-risk residents in ERFLs planning.

Based on the evolution of different generations shown in Fig. 7, we can find that the improvement of non-dominated solutions becomes less and less until a convergence is reached (Fig. 7a). Meanwhile, the NSGA-II is able to maintain a better diversity of nondominated solutions across the three objectives during the iterations (Fig. 7b).

Figure $8 \mathrm{~b}$ exhibits a similar trend as Fig. 7a regarding the improvement of non-dominated solutions, indicating the performance of convergence during the iterations. In Fig. $8 \mathrm{~b}$, the convergence metric quickly moves to nearly zero, implying that the nondominated solutions starting from a random set quickly approached the final Pareto-optimal frontier. After about 30 generations, the solutions come very close to the final Paretooptimal frontier, clearly demonstrating the global optimal searching ability of the model. From the 30th generation to the 100th generation, changes in the convergence metric are not obvious, which indicates that the values of objective functions are not significantly changed. However, in the meantime, the diversity of non-dominated solutions increases from generation to generation as presented in Fig. 8a, which can be validated by the 3D plot of the evolutionary process shown in Fig. 7b. All the results presented in Figs. 7 and 8 provide evidence that the NSGA-II algorithm coupled with the designed spatial representation and encoding strategy can successfully generate Pareto-optimal frontier for the multi-objective ERFLs optimization model constructed in this study.

The candidate locations of solution "A" concentrate around the centroid of the risk map, because service capacity and distance of each facility are not limited in objective function Obj1. For a certain facility, this means that the closer its location to the centroid of the risk map, the more amount of risk demands it will cover. The spatial pattern of solution "B" arises because of the weight value $R_{i}$ involved in the objective function Obj2, which represents the risk value of each mesh. The spatial pattern of solution " $\mathrm{C}$ " comes about 
(a)
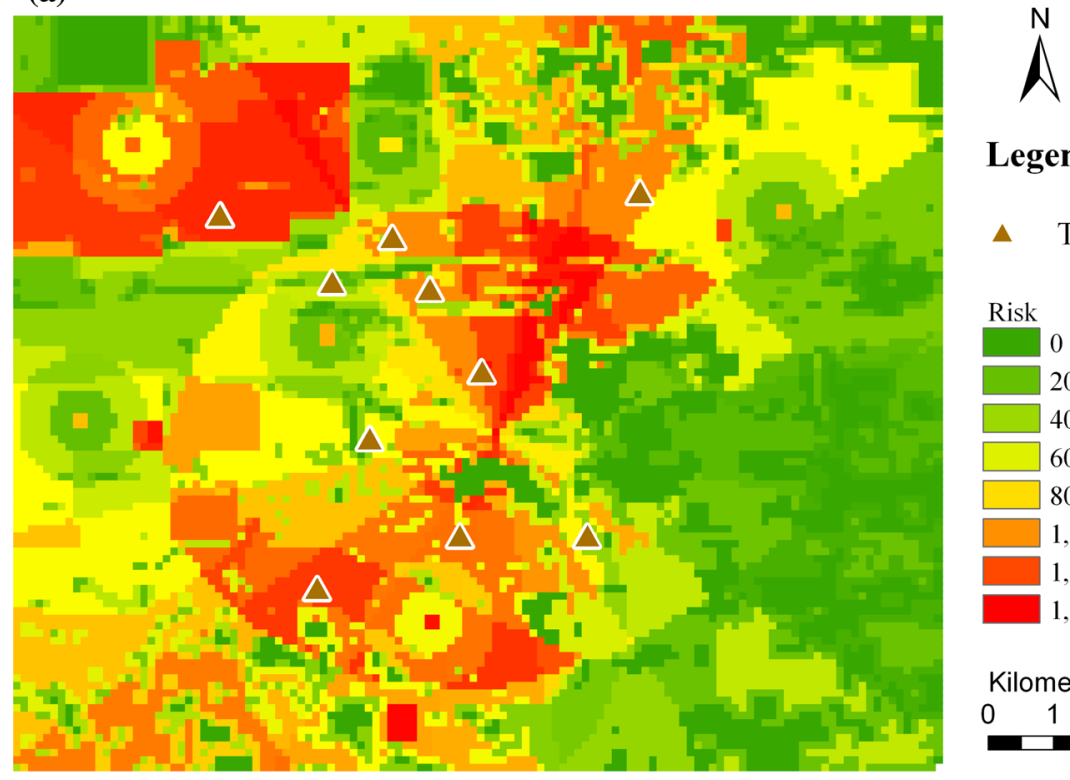

\section{Legend}

- Trade-Off

Risk

$0-200$
$200-400$
$400-600$
$\square 00-800$
$\square$
$\square 00-1,000$
$1,000-1,200$
$1,200-1,400$
$1,400-1,600$

Kilometers

\begin{tabular}{lll}
0 & 1 & 2 \\
\hline
\end{tabular}

(b)

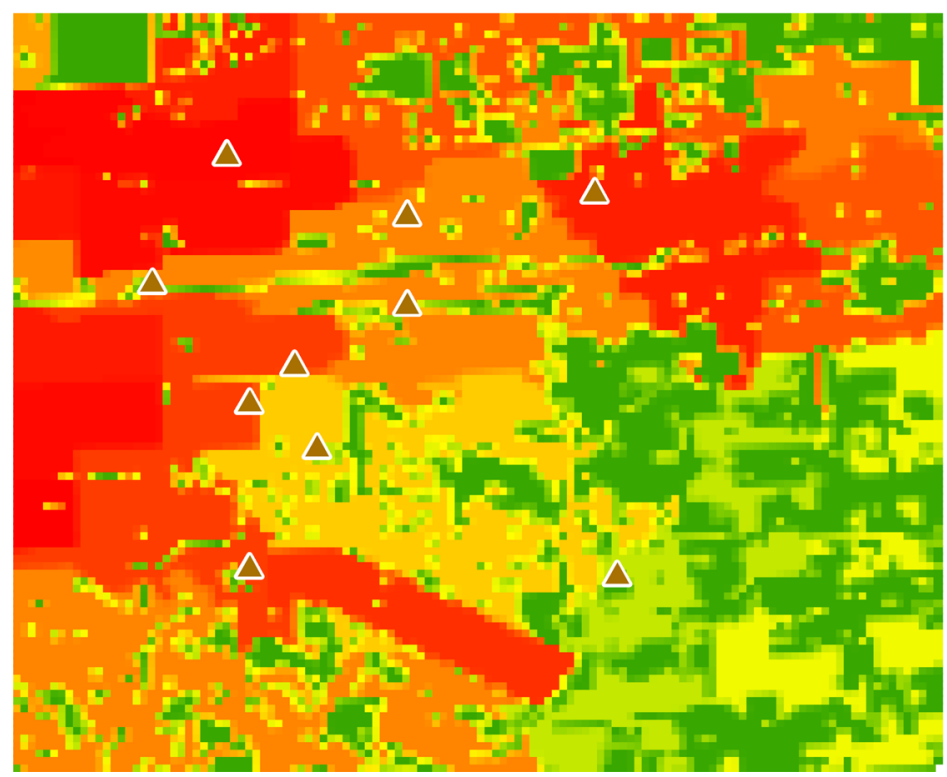

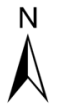

Legend

$\Delta \quad$ Trade-Off

Population Density (persons $/ \mathrm{km}^{2}$ )

$0-5,000$ $5,000-10,000$ $10,000-15,000$ $15,000-20,000$ $20,000-25,000$ $25,000-30,000$ $30,000-35,000$ $35,000-40,000$ $40,000-45,000$ Kilometers

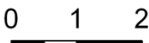

Fig. 11 Representative Trade-off results of a risk-based model and $\mathbf{b}$ population-based model

because of the emphasis of minimizing the maximum distance between any mesh and its closest facility in order to achieve equity.

According to Table 1, each objective-preferred solution tends to be extreme, but they definitely reach the best scores with respect to the preferential single objective. Moreover, 
the "Trade-Off" scenario has the most balanced values of all the three objectives. The location maps and the attributes information table demonstrate the effectiveness of the multi-objective spatial optimization model constructed as well as the model solution method proposed in this study.

The comparison result of risk-based model (Fig. 11a) and population-based model (Fig. 11b) shows that risk mapping has significant effect on the locations of optimal emergency rescue facilities. Therefore, the involvement of risk-based approach into solving the ERFLs optimization problem is necessary. The risk-based optimal ERFLs map could potentially enhance rescue capability and improve public safety.

\section{Conclusions}

This study presents an innovational methodological framework that combines risk analysis and mapping with a multi-objective optimization model for identifying locations of emergency rescue facilities in the context of urban environmental accidents. With an appropriate spatial representation and encoding strategy designed, NSGA-II algorithm is used for model solution. A case study is presented to demonstrate the proposed methodology. The results of risk mapping show that it provides a new and comprehensive perspective for guiding emergency rescue planning, as it reflects not only the potential exposure of residents to the hazard, but also the likely rescue costs for the residents. Moreover, the optimization results provide evidences that the model can successfully capture a pool of alternative non-dominated solutions for the ERFLs problem, and the involvement of risk mapping has significant effect on the locations of emergency rescue facilities. The findings show that the methodology proposed in this study provides an interesting perspective to solve ERFLs problem, and it has the potential to be a useful decision support tool for urban emergency management and planning.

Although accurate risk value of each mesh can not be calculated by the semi-quantitative risk assessment approach used in this study, the relative risk level between meshes can be determined to support delineating the potential hazard of environmental accidents to the adjacent urban areas, which is enough to meet the need of ERFLs problem. In our future research, some kinds of quantitative risk assessment methods will be involved to improve the semi-quantitative method. Moreover, the entire study region is assumed to be continuous space in this study; however, urban area is usually discrete space. Therefore, the Euclidean distance used in the model assumption may not be feasible in some real applications, and improvement in the calculation of spatial distance by replacing Euclidean distance with the actual transport network distance is necessary in the future research. Although the hazard source is fictive and only toxic release accident scenario is considered in this study, its generic formulation allows applying it to an actual urban system with the configurations of any multiple, fixed or mobile hazard sources.

Acknowledgments We appreciate the anonymous reviewers for their insightful comments.

\section{Appendix}

See Table 6. 
Table 6 Statistical analysis results of final Pareto-optimal solutions in each run

\begin{tabular}{|c|c|c|c|c|c|c|c|}
\hline No. of run & Num. & Objectives & Max & Min & Average & $\begin{array}{l}\text { Average } \\
\text { (normalization) }\end{array}$ & Time (min) \\
\hline \multirow[t]{4}{*}{1} & 680 & Obj1 & 32.435485 & 28.093668 & 29.508874 & 0.325948 & 125.80 \\
\hline & & Obj2 & 79.058380 & 34.254638 & 49.716910 & 0.345111 & \\
\hline & & Obj3 & 42.880926 & 15.135777 & 28.398469 & 0.478018 & \\
\hline & & sum & & & & 1.149077 & \\
\hline \multirow[t]{4}{*}{2} & 676 & Obj1 & 32.300466 & 28.092472 & 29.597232 & 0.357596 & 122.70 \\
\hline & & Obj2 & 78.251869 & 34.615320 & 48.216484 & 0.311692 & \\
\hline & & Obj3 & 43.261128 & 15.248167 & 29.150748 & 0.496291 & \\
\hline & & sum & & & & 1.165579 & \\
\hline \multirow[t]{4}{*}{3} & 750 & Obj1 & 32.624406 & 28.113063 & 29.497709 & 0.306925 & 124.00 \\
\hline & & Obj2 & 79.757743 & 33.765028 & 49.072907 & 0.332833 & \\
\hline & & Obj3 & 41.946115 & 15.384241 & 28.745361 & 0.503019 & \\
\hline & & sum & & & & 1.142777 & \\
\hline \multirow[t]{4}{*}{4} & 609 & Obj1 & 32.901927 & 28.118435 & 29.708752 & 0.332459 & 113.75 \\
\hline & & Obj2 & 75.676183 & 33.868759 & 47.087620 & 0.316185 & \\
\hline & & Obj3 & 43.261128 & 14.530745 & 27.145029 & 0.439057 & \\
\hline & & sum & & & & 1.087701 & \\
\hline \multirow[t]{4}{*}{5} & 725 & Obj1 & 32.033271 & 28.113810 & 29.574208 & 0.372602 & 118.13 \\
\hline & & Obj2 & 79.454041 & 35.255729 & 49.591637 & 0.324354 & \\
\hline & & Obj3 & 41.175203 & 16.321472 & 27.248492 & 0.439653 & \\
\hline & & sum & & & & 1.136609 & \\
\hline \multirow[t]{4}{*}{6} & 648 & Obj1 & 32.623817 & 28.172540 & 29.837407 & 0.374020 & 122.97 \\
\hline & & Obj2 & 76.086876 & 34.065091 & 46.232968 & 0.289561 & \\
\hline & & Obj3 & 41.309857 & 15.542712 & 26.639741 & 0.430666 & \\
\hline & & sum & & & & 1.094247 & \\
\hline \multirow[t]{4}{*}{7} & 769 & Obj1 & 32.467122 & 28.159695 & 29.780319 & 0.376239 & 127.88 \\
\hline & & $O b j 2$ & 74.262748 & 33.997573 & 45.498940 & 0.285641 & \\
\hline & & Obj3 & 43.937395 & 15.384241 & 26.806288 & 0.400028 & \\
\hline & & sum & & & & 1.061908 & \\
\hline \multirow[t]{4}{*}{8} & 617 & Obj1 & 33.005322 & 28.169573 & 29.603295 & 0.296484 & 123.08 \\
\hline & & Obj2 & 74.070372 & 34.912708 & 47.646429 & 0.325191 & \\
\hline & & Obj3 & 41.946115 & 14.404839 & 27.711914 & 0.483168 & \\
\hline & & sum & & & & 1.104843 & \\
\hline \multirow[t]{4}{*}{9} & 729 & Obj1 & 33.148225 & 28.126663 & 29.642878 & 0.301941 & 132.77 \\
\hline & & Obj2 & 80.531788 & 34.872954 & 48.794369 & 0.304901 & \\
\hline & & Obj3 & 43.597709 & 14.604340 & 28.184529 & 0.468389 & \\
\hline & & sum & & & & 1.075231 & \\
\hline \multirow[t]{4}{*}{10} & 615 & Obj1 & 32.308273 & 28.089406 & 29.557327 & 0.347942 & 132.52 \\
\hline & & Obj2 & 81.324301 & 34.721276 & 48.692603 & 0.299794 & \\
\hline & & Obj3 & 42.926459 & 15.470331 & 27.424897 & 0.435406 & \\
\hline & & sum & & & & 1.083142 & \\
\hline
\end{tabular}

Num., total number of final Pareto-optimal solutions in each run; Max/Min, maximum/ minimum value of each objective function in each run; Average/Average (Normalization), average value of each objective function before/ after being normalized in each run; Sum, sum of the average normalized values of the three-objective functions in each run; Time (minutes), the computing time of each run 


\section{References}

AEGL Co (2009) AEGL Program. http://www.epa.gov/oppt/aegl/

Alçada-Almeida L, Tralhão L, Santos L, Coutinho-Rodrigues J (2009) A multiobjective approach to locate emergency shelters and identify evacuation routes in urban areas. Geogr Anal 41(1):9-29. doi:10.1111/ j.1538-4632.2009.00745.x

Ale BJM (2002) Risk assessment practices in The Netherlands. Saf Sci 40(1-4):105-126. doi:10.1016/ S0925-7535(01)00044-3

AQSIQ (2009) Identification of major hazard installations for dangerous chemicals, GB18218-2009. http:// www.jssrcc.com.cn/list.asp?id=533

Basta C, Neuvel JMM, Zlatanova S, Ale B (2007) Risk-maps informing land-use planning processes: a survey on the Netherlands and the United Kingdom recent developments. J Hazard Mater 145(1-2):241-249. doi:10.1016/j.jhazmat.2006.11.032

Bell JE, Griffis SE, Cunningham WA, Eberlan JA (2011) Location optimization of strategic alert sites for homeland defense. Omega 39(2):151-158. doi:10.1016/j.omega.2010.05.004

Bhattacharya R, Bandyopadhyay S (2010) Solving conflicting bi-objective facility location problem by NSGA II evolutionary algorithm. Int J Adv Manuf Technol 51(1-4):397-414. doi:10.1007/s00170010-2622-6

Bottelberghs PH (2000) Risk analysis and safety policy developments in the Netherlands. J Hazard Mater 71(1-3):59-84. doi:10.1016/S0304-3894(99)00072-2

Cao K, Batty M, Huang B, Liu Y, Yu L, Chen JF (2011) Spatial multi-objective land use optimization: extensions to the non-dominated sorting genetic algorithm-II. Int J Geogr Inf Sci 25(12):1949-1969. doi:10.1080/13658816.2011.570269

Caunhye AM, Nie X, Pokharel S (2012) Optimization models in emergency logistics: a literature review. Socio-Econ Plan Sci 46(1):4-13. doi:10.1016/j.seps.2011.04.004

Chrissis J (1980) Locating emergency service facilities in a developing area. Fire Technol 16(1):63-69. doi:10.1007/BF01857844

Church RL, Murray AT (2009) Business site selection, location analysis, and GIS. Wiley, Hoboken

Church R, ReVelle C (1974) The maximal covering location problem. Pap Reg Sci As 32(1):101-118. doi:10.1007/BF01942293

Coello Coello CA (1999) A comprehensive survey of evolutionary-based multiobjective optimization techniques. Knowl Inf Syst 1(3):269-308. doi:10.1007/BF03325101

Coello Coello CA (2005) Recent trends in evolutionary multiobjective optimization. In: Abraham A, Jain L, Goldberg R (eds) Evolutionary multiobjective optimization. Advanced information and knowledge processing. Springer, London, pp 7-32. doi:10.1007/1-84628-137-7-2

Cozzani V, Bandini R, Basta C, Christou MD (2006) Application of land-use planning criteria for the control of major accident hazards: a case-study. J Hazard Mater 136(2):170-180. doi:10.1016/ j.jhazmat.2005.12.031

CPR18E (1999) Guidelines for quantitative risk assessment: purple book. Directorate-General for Social Affairs and Employment, The Hague

Cutter SL, Mitchell JT, Scott MS (2000) Revealing the vulnerability of people and places: a case study of Georgetown County, South Carolina. Ann Assoc Am Geogr 90(4):713-737. doi:10.1111/0004-5608. 00219

Daskin MS (1995) Network and discrete location: models, algorithms, and applications. Wiley, London

Deb K, Pratap A, Agarwal S, Meyarivan T (2002) A fast and elitist multiobjective genetic algorithm: NSGA-II. IEEE Trans Evol Comput 6(2):182-197. doi:10.1109/4235.996017

EPA U, NOAA (2007) ALOHA user's manual. http://response.restoration.noaa.gov/aloha

Fan B (2014) Hybrid spatial data mining methods for site selection of emergency response centers. Nat Hazards 70(1):643-656. doi:10.1007/s11069-013-0833-5

Farhana B, Murray AT (2008) Siting park-and-ride facilities using a multi-objective spatial optimization model. Comput Oper Res 35(2):445-456. doi:10.1016/j.cor.2006.03.009

FEMA (2008) IS-26 Guide to points of distribution. http://training.fema.gov/EMIWeb/IS/IS26.asp

Georgiadou PS, Papazoglou IA, Kiranoudis CT, Markatos NC (2010) Multi-objective evolutionary emergency response optimization for major accidents. J Hazard Mater 178(1-3):792-803. doi:10.1016/j. jhazmat.2010.02.010

Horner MW, Downs JA (2010) Optimizing hurricane disaster relief goods distribution: model development and application with respect to planning strategies. Disasters 34(3):821-844. doi:10.1111/.1467-7717. 2010.01171.x

Huang B, Fery P, Xue L, Wang Y (2008) Seeking the Pareto front for multiobjective spatial optimization problems. Int J Geogr Inf Sci 22(5):507-526. doi:10.1080/13658810701492365 
Huang L, Wan W, Li F, Li B, Yang J, Bi J (2011) A two-scale system to identify environmental risk of chemical industry clusters. J Hazard Mater 186(1):247-255. doi:10.1016/j.jhazmat.2010.10.117

Indriasari V, Mahmud AR, Ahmad N, Shariff ARM (2010) Maximal service area problem for optimal siting of emergency facilities. Int J Geogr Inf Sci 24(2):213-230

Jia HZ, Ordonez F, Dessouky M (2007a) A modeling framework for facility location of medical services for large-scale emergencies. IIE Trans 39(1):41-55. doi:10.1080/07408170500539113

Jia HZ, Ordonez F, Dessouky MM (2007b) Solution approaches for facility location of medical supplies for large-scale emergencies. Comput Ind Eng 52(2):257-276. doi:10.1016/j.cie.2006.12.007

Jian-wei G, Xiao-ping Z, Ying W (2012) Environmental hazards analysis and discussion on ammonia tank leakage in power plant denitrification projects. J Saf Environ 12(6):108-112

Jonkman SN, van Gelder PHAJM, Vrijling JK (2003) An overview of quantitative risk measures for loss of life and economic damage. J Hazard Mater 99(1):1-30. doi:10.1016/S0304-3894(02)00283-2

Knowles J, Corne D, Deb K (2008) Multiobjective problem solving from nature. Springer, New York

Kontić D, Kontić B (2009) Introduction of threat analysis into the land-use planning process. J Hazard Mater 163(2-3):683-700. doi:10.1016/j.jhazmat.2008.07.040

Lančinskas A, Żilinskas J (2013) Solution of multi-objective competitive facility location problems using parallel NSGA-II on large scale computing systems. In: Manninen P, Öster P (eds) Applied parallel and scientific computing, vol 7782. Lecture notes in computer science. Springer, Berlin, pp 422-433. doi:10.1007/978-3-642-36803-5_31

Lara-Valencia F, Harlow SD, Lemos MC, Denman CA (2009) Equity dimensions of hazardous waste generation in rapidly industrialising cities along the United States-Mexico border. J Environ Plan Manag 52(2):195-216. doi:10.1080/09640560802666545

Lee G, Xiao N (2009) Examining the tradeoff between residential broadband service coverage and network connectivity using a bi-objective facility location model*. Pap Reg Sci 88(3):547-562. doi:10.1111/j. 1435-5957.2008.00206.x

Lee YM, Ghosh S, Ettl M (2009) Simulating distribution of emergency relief supplies for disaster response operations. In: Proceedings of winter simulation conference, pp 2657-2668

Li X, Yeh AGO (2005) Integration of genetic algorithms and GIS for optimal location search. Int J Geogr Inf Sci 19(5):581-601. doi:10.1080/13658810500032388

Li F, Bi J, Huang L, Qu C, Yang J, Bu Q (2010) Mapping human vulnerability to chemical accidents in the vicinity of chemical industry parks. J Hazard Mater 179(1-3):500-506. doi:10.1016/j.jhazmat.2010.03. 031

Liu N, Huang B, Chandramouli M (2006) Optimal siting of fire stations using GIS and ANT algorithm. J Comput Civil Eng 20(5):361-369. doi:10.1061/(Asce)0887-3801(2006)20:5(361

Maliszewski PJ, Horner MW (2010) A spatial modeling framework for siting critical supply infrastructures. Prof Geographer 62(3):426-441. doi:10.1080/00330121003788408

Maliszewski PJ, Kuby MJ, Horner MW (2012) A comparison of multi-objective spatial dispersion models for managing critical assets in urban areas. Comput Environ Urban Syst 36(4):331-341. doi:10.1016/j. compenvurbsys.2011.12.006

Miettinen K (1999) Nonlinear multiobjective optimization. Kluwer, Boston

Mohammadi N, Malek MR, Alesheikh AA (2010) A new GA based solution for capacitated multi source Weber problem. Int J Comput Intel Syst 3(5):514-521. doi:10.1080/18756891.2010.9727718

Murray A (2010) Advances in location modeling: GIS linkages and contributions. J Geogr Syst 12(3):335-354. doi:10.1007/s10109-009-0105-9

Planas E, Arnaldos J, Silvetti B, Vallée A, Casal J (2006) A Risk Severity Index for industrial plants and sites. J Hazard Mater 130(3):242-250. doi:10.1016/j.jhazmat.2005.07.015

Revelle C, Hogan K (1989) The maximum reliability location problem and $\alpha$-reliablep-center problem: derivatives of the probabilistic location set covering problem. Ann Oper Res 18(1):155-173. doi:10. 1007/BF02097801

Revelle C, Snyder S (1995) Integrated fire and ambulance siting: a deterministic model. Socio-Econ Plan Sci 29(4):261-271. doi:10.1016/0038-0121(95)00014-3

Salvi O, Debray B (2006) A global view on ARAMIS, a risk assessment methodology for industries in the framework of the SEVESO II directive. J Hazard Mater 130(3):187-199. doi:10.1016/j.jhazmat.2005. 07.034

Sebos I, Progiou A, Symeonidis P, Ziomas I (2010) Land-use planning in the vicinity of major accident hazard installations in Greece. J Hazard Mater 179(1-3):901-910. doi:10.1016/j.jhazmat.2010.03.091

Srinivas N, Deb K (1994) Muiltiobjective optimization using nondominated sorting in genetic algorithms. Evol Comput 2(3):221-248. doi:10.1162/evco.1994.2.3.221 
Tena-Chollet F, Tixier J, Dusserre G, Mangin JF (2013) Development of a spatial risk assessment tool for the transportation of hydrocarbons: methodology and implementation in a geographical information system. Environ Model Softw 46:61-74. doi:10.1016/j.envsoft.2013.02.010

Tixier J, Dandrieux A, Dusserre G, Bubbico R, Mazzarotta B, Silvetti B, Hubert E, Rodrigues N, Salvi O (2006) Environmental vulnerability assessment in the vicinity of an industrial site in the frame of ARAMIS European project. J Hazard Mater 130(3):251-264. doi:10.1016/j.jhazmat.2005.07.020

Tong D, Murray AT (2012) Spatial optimization in geography. Ann As Am Geogr 102(6):1290-1309. doi:10.1080/00045608.2012.685044

Tong D, Murray A, Xiao N (2009) Heuristics in spatial analysis: a genetic algorithm for coverage maximization. Ann As Am Geogr 99(4):698-711. doi:10.1080/00045600903120594

Wu XL, Murray AT, Xiao NC (2011) A multiobjective evolutionary algorithm for optimizing spatial contiguity in reserve network design. Landsc Ecol 26(3):425-437. doi:10.1007/s10980-011-9571-9

Xiang W-N, Stuber RMB, Meng X (2011) Meeting critical challenges and striving for urban sustainability in China. Landsc Urban Plan 100(4):418-420. doi:10.1016/j.landurbplan.2011.02.018

Xiao N (2008) A unified conceptual framework for geographical optimization using evolutionary algorithms. Ann Assoc Am Geogr 98(4):795-817. doi:10.1080/00045600802232458

Xiao N, Bennett DA, Armstrong MP (2002) Using evolutionary algorithms to generate alternatives for multiobjective site-search problems. Environ Plan A 34(4):639-656. doi:10.1068/a34109

Xiao N, Bennett DA, Armstrong MP (2007) Interactive evolutionary approaches to multiobjective spatial decision making: a synthetic review. Comput Environ Urban Syst 31(3):232-252. doi:10.1016/j. compenvurbsys.2006.08.001

Zhou Y, Liu M (2012) Risk assessment of major hazards and its application in urban planning: a case study. Risk Anal 32(3):566-577. doi:10.1111/j.1539-6924.2011.01670.x

Zitzler E, Thiele L (1999) Multiobjective evolutionary algorithms: a comparative case study and the Strength Pareto approach. IEEE Trans Evol Comput 3(4):257-271. doi:10.1109/4235.797969 